\title{
Reflection high-energy electron diffraction analysis of polycrystalline films with grain size and orientation distributions
}

\author{
J. W. Hartman, R. T. Brewer, ${ }^{\text {a) }}$ and Harry A. Atwater \\ Thomas J. Watson Laboratory of Applied Physics, California Institute of Technology, Pasadena, \\ California 91125
}

(Received 24 June 2002; accepted 5 August 2002)

\begin{abstract}
We report a computationally efficient algorithm to calculate reflection high-energy electron diffraction (RHEED) intensities from well-textured, small-grained polycrystalline films in the kinematic limit. We also show how the intensity maps of the spots in a RHEED pattern from such a film can be quantitatively analyzed to determine the film's average grain size, as well as its in-plane orientation and texture distributions. We find that the in-plane orientation and texture distribution widths of these films can be determined to within 1 degree and that the average lateral grain size can be measured to within a fraction of a nanometer after suitable calibration of our technique. (c) 2002 American Institute of Physics. [DOI: 10.1063/1.1510954]
\end{abstract}

\section{INTRODUCTION}

The ability to measure grain size, crystallographic texture, and surface roughness during deposition of thin polycrystalline and single crystal films is of fundamental interest and commercial importance. Reflection high-energy electron diffraction (RHEED) is a measurement technique which is compatible with the deposition conditions of many high vacuum deposition techniques (e.g., sputter deposition, molecular beam epitaxy) but is typically used only to monitor surface roughness and surface structure during the growth of single crystal materials because quantitative interpretation of the RHEED intensities is difficult. Recently, models which are valid in the limit of very large grains ${ }^{1,2}$ have been developed to treat grain orientation distributions in polycrystalline materials and have been used to determine out-of-plane texture for a CoCr alloy. ${ }^{3}$ We have developed a fast algorithm able to calculate RHEED intensities from well-textured, narrow in-plane oriented, and small-grained polycrystalline films. The algorithm enables us to determine the effects of distributions in texture, in-plane orientation, and grain size upon the RHEED pattern. Evaluation of the algorithm is fast enough to permit an investigator to use standard fitting procedures (search algorithms) to fit an observed RHEED pattern to the distribution parameters. Alternatively, our algorithm permits one to easily develop "look-up" tables which relate simple parameters describing an observed diffraction pattern to parameters describing the distribution of grain size, texture, and in-plane orientation. Evaluation time is linear in the number of pixels and requires a few seconds to evaluate a diffraction pattern with $640 \times 480$ resolution on a $350 \mathrm{MHz}$ Pentium II coprocessor.

\footnotetext{
a) Author to whom correspondence should be addressed; http:// daedalus.caltech.edu/ rhett/index.shtml; 626-395-2193; electronic mail: rhett@its.caltech.edu
}

\section{MODEL}

\section{A. Basics}

In the kinematic approximation, scattering from a periodic potential (e.g., atoms in a crystal lattice) is elastic and related to the Fourier transform (FT) of the scattering potential. ${ }^{4,5}$ The amplitude for scattering an initial wave vector $k \hat{e}$ into a final wave vector $k \hat{n}$ is proportional to the FT of the interaction potential at the wave vector $k(\hat{n}-\hat{e})$ :

$$
A(k \hat{n} \rightarrow k \hat{e}) \propto-i \int d^{3} r \exp \left[-i k(\hat{n}-\hat{e})^{T} \vec{r}\right] V(\vec{r}),
$$

where $V(\vec{r})$ is the periodic potential describing interaction of the electrons with the atoms in the scattering target. The probability of scattering $k \hat{e}$ into $k \hat{n}$ is equal to the square of the amplitude. For later notational convenience, we define the interior and exterior products between two vectors as

$$
\vec{k}^{T} \vec{r} \equiv k_{x} x+k_{y} y+k_{z} z
$$

and

$$
\vec{r} \vec{k}^{T} \equiv\left(\begin{array}{ccc}
x k_{x} & x k_{y} & x k_{z} \\
y k_{x} & y k_{y} & y k_{z} \\
z k_{x} & z k_{y} & z k_{z}
\end{array}\right) .
$$

The scattering potential for an infinite, perfectly periodic crystal may be written

$$
V(\vec{r})=\sum_{\vec{R}} \nu(\vec{r}-\vec{R})=\sum_{\vec{G}} V_{\vec{G}} \exp \left(i \vec{G}^{T} \vec{r}\right),
$$

where $\{\vec{R}\}$ are the Bravais lattice vectors describing the positions of the target atoms and $\{\vec{G}\}$ are the reciprocal lattice vectors. The vectors $\{\vec{G}\}$ satisfy $\vec{G}^{T} \vec{R} \bmod 2 \pi=0$ for all lattice vectors $\vec{R}$. In a perfect infinite crystal, scattering can occur only if the momenta of the incident and scattered beams differ by a reciprocal lattice vector of the crystal. The scattering potential of a polycrystalline target composed of 
many finite sized grains with slightly different texture orientations may be developed from the perfect crystal potential by introducing grain "shape" envelope functions and rotations of the reciprocal lattice vectors in each of the finite sized grains: ${ }^{4,5}$

$$
V(\vec{r})=\sum_{g} \Theta_{g}\left(\vec{r}-\vec{r}_{g}\right) \sum_{\vec{G}} V_{\vec{G}} \exp \left[i\left(\mathbf{B}_{g} \vec{G}\right)^{T}\left(\vec{r}-\vec{a}_{g}\right)\right],
$$

where $g$ indexes the individual grains, $\left\{\Theta_{g}\left(\vec{r}-\vec{r}_{g}\right)\right\}$ are the envelope functions describing the shape and location of the individual grains:

$$
\Theta_{g}\left(\vec{r}-\vec{r}_{g}\right)=\left\{\begin{array}{c}
1 \vec{r} \text { inside grain boundary } \\
0 \vec{r} \text { outside grain boundary }
\end{array},\right.
$$

$\left\{\vec{r}_{g}\right\}$ are the centroids of the grains, $\left\{\mathbf{B}_{g}\right\}$ are matrices describing the texture rotation of the individual grains relative to some global coordinate system, and $\left\{\vec{a}_{g}\right\}$ describe displacements between grain lattice positions that may be finite even between grains with no relative texture difference. Both the distribution of textures in the ensemble of grains on the thin film and the finite grain size will broaden the distribution of allowed scattering momentum eigenstates.

Given the polycrystalline form of the scattering potential [Eq. (4)] the scattering amplitude [Eq. (1)] may be written

$$
A(k \hat{n} \rightarrow k \hat{e}) \propto \sum_{\vec{G}, g} \exp \left(i \phi_{\vec{G}, g}\right) A_{\vec{G}, g},
$$

where

$$
\phi_{\vec{G}, g}=\left(\mathbf{B}_{g} \vec{G}\right)^{T}\left(\vec{r}_{g}-\vec{a}_{g}\right)-k(\hat{n}-\hat{e})^{T} \vec{r}_{g}
$$

and

$$
A_{\vec{G}, g}=-i V_{\vec{G}} \int d^{3} r \exp \left[i\left(\mathbf{B}_{g} \vec{G}-k(\hat{n}-\hat{e})\right)^{T} \vec{r}\right] \Theta_{g}(\vec{r}) .
$$

We have separated the scattering amplitude into phase components $\phi_{\vec{G}, g}$ which are expected to vary strongly and randomly from grain to grain, and amplitude components $A_{\vec{G}, g}$ which depend on the individual grain sizes, shapes, and orientations.

\section{B. Random phase approximation}

Ultimately, we are interested in the scattering transition probabilities, which are derived from the scattering amplitudes as

$$
P(k \hat{e} \rightarrow k \hat{n})=|A(k \hat{e} \rightarrow k \hat{n})|^{2} .
$$

In our model we note that rapid variations in the phase terms of Eqs. (6) and (7) allow us to calculate the probability for scattering using the random phase approximation. That is

$$
\begin{aligned}
P(k \hat{e} \rightarrow k \hat{n}) & =\sum_{(\vec{G}, g)\left(\vec{G}^{\prime}, g^{\prime}\right)} \exp \left[i\left(\phi_{\vec{G}, g}-\phi_{\vec{G}^{\prime}, g^{\prime}}\right)\right] A_{\vec{G}, g^{\prime}} \bullet A_{\vec{G}^{\prime}, g^{\prime}}^{*} \\
& \doteq \sum_{\vec{G}, g}\left|A_{\vec{G}, g}\right|^{2}
\end{aligned}
$$

where $A_{\vec{G}^{\prime}, g^{\prime}}^{*}$ is the complex conjugate of $A_{\vec{G}^{\prime}, g^{\prime}}$ and we have used the fact that the cross terms, which satisfy $(\vec{G}, g) \neq\left(\vec{G}^{\prime}, g^{\prime}\right)$, have a random phase and so their sum self averages to zero. In the development of our model we find that it is advantageous to leave the individual scattering amplitudes $A_{\vec{G}, g}$ in integral form. Then, redefining the integration variables in the double integral that results from writing $\left|A_{\vec{G}, g}\right|^{2}$, the scattering probability is

$$
\begin{aligned}
P(k \hat{e} \rightarrow k \hat{n}) \propto & \sum_{\vec{G}, g}\left|V_{\vec{G}}\right|^{2} \int d^{3} r \exp \left[i\left(\mathbf{B}_{g} \vec{G}-k(\hat{n}-\hat{e})^{T} \vec{r}\right)\right] \\
& \times \int d^{3} r^{\prime} \Theta_{g}\left(\vec{r}+\vec{r}^{\prime}\right) \Theta_{g}\left(\vec{r}^{\prime}\right) .
\end{aligned}
$$

The sum over individual grains $\Sigma_{g}(\ldots)$ may be replaced by an average over the distribution of grains. If we assume that grain shape and texture are independent, then we may replace $\Sigma_{g}(\ldots)$ by the product of independent averages over grain texture and size

$$
\begin{aligned}
P(k \hat{e} \rightarrow k \hat{n}) \propto & \sum_{\vec{G}}\left|V_{\vec{G}}\right|^{2} \int d^{3} r \exp \left[-i k(\hat{n}-\hat{e})^{T} \vec{r}\right] \\
& \times\left\langle\exp \left[i\left(\mathbf{B}_{g} \vec{G}\right)^{T} \vec{r}\right]\right\rangle_{\text {texture }} \\
& \times\left\langle\int d^{3} r^{\prime} \Theta_{g}\left(\vec{r}+\vec{r}^{\prime}\right) \Theta_{g}\left(\vec{r}^{\prime}\right)\right\rangle_{\text {size }} .
\end{aligned}
$$

\section{Grain shape function}

Consider now evaluation of the shape convolution function which appears in Eq. (12)

$$
T_{g}(\vec{r})=\int d^{3} r^{\prime} \Theta_{g}\left(\vec{r}+\vec{r}^{\prime}\right) \Theta_{g}\left(\vec{r}^{\prime}\right) .
$$

The simplest shape function for a grain of lateral size $L_{g}$ and height $h$ normal to the film surface is a square tile with finite thickness

$$
\Theta_{g}(x, y, z)=\left\{\begin{array}{cc}
1 & \left|\frac{x}{L_{g}}\right|,\left|\frac{y}{L_{g}}\right|,\left|\frac{z}{h}\right|<0.5 \\
0 & \text { otherwise }
\end{array} .\right.
$$

In this case, self convolution of the grain shape function is trivial

$$
T_{g}(x, y, z)=\left\{\begin{array}{ll}
L_{g}^{2} h\left(1-\left|\frac{x}{L_{g}}\right|\right)\left(1-\left|\frac{y}{L_{g}}\right|\right)\left(1-\left|\frac{z}{h}\right|\right) & \left|\frac{x}{L_{g}}\right|,\left|\frac{y}{L_{g}}\right|,\left|\frac{z}{h}\right|<1 \\
0 & \text { otherwise }
\end{array}\right\} .
$$


This function is sharply peaked near the origin, decays rapidly to zero, and may be approximated very well by a Gaussian with a properly chosen width

$$
T_{g}(\vec{r})=\frac{L_{g}^{2} h}{\left(2 \pi \sigma^{2}\right)^{3 / 2}} \exp \left(-\frac{1}{2} \vec{r}^{T} \Sigma \vec{r}\right)
$$

where

$$
\Sigma=\left(\begin{array}{ccc}
1 /\left(\sigma L_{g}\right)^{2} & 0 & 0 \\
0 & 1 /\left(\sigma L_{g}\right)^{2} & 0 \\
0 & 0 & 1 /(\sigma h)^{2}
\end{array}\right)
$$

Here $\sigma=0.435$ and we have defined the "grain shape matrix" $\Sigma$ for later convenience. In RHEED, the effective grain height $h$ is determined by extinction of the primary electron beam as it penetrates the target; for grazing incidence, the effective scattering depths may be on the order of nanometers. One can estimate $h$ by considering the angle of incidence and the electron mean free path $\lambda$ in the target: $h$ $\sim \lambda \sin \theta_{\text {inc }}$. Surface roughness will expose more Bravais lattice planes and increase the effective scattering depth. Hence the effective penetration depth is a function of both the primary beam extinction coefficient in the dense material and the roughness of the surface.

The effective scattering depth should, in general, be independent of the lateral grain size. Performing the full "size" average over all grain sizes to calculate the averaged shape convolution function $T(\vec{r})=\left\langle T_{g}(\vec{r})\right\rangle_{\text {size }}$ will, to first order replace $L_{g}$ in Eq. (16) by an effective lateral grain size representative of the entire grain size distribution. Thus, we approximate

$$
\left\langle\int d^{3} r^{\prime} \Theta_{g}\left(\vec{r}+\vec{r}^{\prime}\right) \Theta_{g}\left(\vec{r}^{\prime}\right)\right\rangle_{\text {size }} \sim \exp \left(-\frac{1}{2} \vec{r}^{T} \sum \vec{r}\right),
$$

where $\Sigma$ is as in Eq. (16), $h$ is the effective penetration depth normal to the film surface which depends on surface roughness and primary beam extinction, and $L_{g}$ is a lateral grain size representative of the distribution of grain sizes.

\section{Grain orientation distribution}

Analytic evaluation of the texture dependent term in Eq. (12) is also possible in the limit of narrow texture distribution. The rotation matrices $\mathbf{B}_{g}$ may be expanded in the smallness of the rotations $\omega_{x}, \omega_{y}$, and $\phi_{z}$, about the three principal axes $\hat{x}, \hat{y}$, and $\hat{z}$ :

$$
\mathbf{B}_{g} \simeq \mathbf{1}+\omega_{x} \mathbf{X}_{x}+\omega_{y} \mathbf{X}_{y}+\phi_{z} \mathbf{X}_{z} \quad \omega_{x}, \omega_{y}, \phi_{z}<10^{\circ},
$$

where $\mathbf{1}$ is the $3 \times 3$ unit matrix and $\mathbf{X}_{x}, \mathbf{X}_{y}$, and $\mathbf{X}_{z}$ are rotation generators about the $\hat{x}, \hat{y}$, and $\hat{z}$ axes

$$
\begin{aligned}
& \mathbf{X}_{x}=\left(\begin{array}{ccc}
0 & 0 & 0 \\
0 & 0 & 1 \\
0 & -1 & 0
\end{array}\right), \mathbf{X}_{y}=\left(\begin{array}{ccc}
0 & 0 & -1 \\
0 & 0 & 0 \\
1 & 0 & 0
\end{array}\right), \\
& \mathbf{X}_{z}=\left(\begin{array}{ccc}
0 & 1 & 0 \\
-1 & 0 & 0 \\
0 & 0 & 0
\end{array}\right) .
\end{aligned}
$$

Under the assumption that the grain textures are normally distributed according to

$$
\begin{aligned}
p\left(\omega_{x}, \omega_{y}, \phi_{z}\right)= & \frac{\exp \left[-\omega_{x}^{2} / 2\left(\Delta \omega_{x}\right)^{2}\right]}{\sqrt{2 \pi\left(\Delta \omega_{x}\right)^{2}}} \frac{\exp \left[-\omega_{y}^{2} / 2\left(\Delta \omega_{y}\right)^{2}\right]}{\sqrt{2 \pi\left(\Delta \omega_{y}\right)^{2}}} \\
& \times \frac{\exp \left[-\phi_{z}^{2} / 2\left(\Delta \phi_{z}\right)^{2}\right]}{\sqrt{2 \pi\left(\Delta \phi_{z}\right)^{2}}},
\end{aligned}
$$

the texture average can be reduced to calculating the Fourier transform of a Gaussian. The exact result may be written

$$
\begin{aligned}
\langle\exp [ & \left.\left.i\left(\mathbf{B}_{g} \vec{G}\right)^{T} \vec{r}\right]\right\rangle_{\text {texture }} \\
= & \exp \left(i \vec{G}^{T} \vec{r}\right) \int d \omega_{x} d \omega_{y} d \phi_{z} p\left(\omega_{x}, \omega_{y}, \phi_{z}\right) \\
& \quad \times \exp \left[i \omega_{x}\left(\mathbf{X}_{x} \vec{G}\right)^{T} \vec{r}+i \omega_{y}\left(\mathbf{X}_{y} \vec{G}\right)^{T} \vec{r}+i \phi_{z}\left(\mathbf{X}_{z} \vec{G}\right)^{T} \vec{r}\right] \\
= & \exp \left[i \vec{G}^{T} \vec{r}-\frac{1}{2} \vec{r}^{T} \Delta_{\vec{G}} \vec{r}\right],
\end{aligned}
$$

where, for notational convenience, we have defined the "texture matrix" which depends on the texture distribution widths and the individual reciprocal lattice vectors

$$
\begin{aligned}
\Delta_{\vec{G}}= & \left(\Delta \omega_{x}\right)^{2} \mathbf{X}_{x} \vec{G}\left(\mathbf{X}_{x} \vec{G}\right)^{T} \\
& +\left(\Delta \omega_{y}\right)^{2} \mathbf{X}_{y} \vec{G}\left(\mathbf{X}_{y} \vec{G}\right)^{T}+\left(\Delta \phi_{z}\right)^{2} \mathbf{X}_{z} \vec{G}\left(\mathbf{X}_{z} \vec{G}\right)^{T} .
\end{aligned}
$$

[See Eq. (2) for definition of the exterior products $(\mathbf{X} \vec{G})$ $\times(\mathbf{X} \vec{G})^{T}$.]

If we substitute Eqs. (21) and (17) into Eq. (12) we see that evaluation of the scattering probability has been reduced to a sum over Fourier transforms of Gaussians. We define $\mathbf{A}_{\vec{G}}^{-1}=\Delta_{\vec{G}}+\boldsymbol{\Sigma}$, perform the integrations, and write the scattering probability in an analytic form as

$$
\begin{aligned}
P(k \hat{e} \rightarrow k \hat{n}) \propto & \sum_{\vec{G}}\left|V_{\vec{G}}\right|^{2} \int d^{3} r \\
& \times \exp \left[i\left(\vec{G}-k(\hat{n}-\hat{e})^{T} \vec{r}\right)\right] \exp \left[-\frac{1}{2} \vec{r}^{T} \mathbf{A}_{\vec{G}}^{-1} \vec{r}\right] \\
& \propto \sum_{\vec{G}}\left|V_{\vec{G}}\right|^{2} \sqrt{\operatorname{det}\left|\mathbf{A}_{\vec{G}}\right|} \exp \left[-\frac{1}{2}(\vec{G}-k(\hat{n}-\hat{e}))^{T}\right. \\
& \left.\times \mathbf{A}_{\vec{G}}(\vec{G}-k(\hat{n}-\hat{e}))\right] .
\end{aligned}
$$

In the kinematic model, the form factors for electronic scattering and $x$-ray scattering from an atom of nuclear charge $Z$ are related by the Mott formula: $\nu_{z}(\vec{G})=(Z$ $\left.-f_{Z}(\vec{G})\right) / G^{2}{ }^{5}$ The x-ray form factors $f_{Z}$ are widely available (see, for example, Ref. 6). Form factors are related to 
the scattering potential by a sum over the basis vectors $(\vec{b})$ describing the positions of atoms in the unit cell of the lattice:

$$
V_{\vec{G}}=\sum_{\vec{b}} \exp \left(i \vec{b}^{T} \vec{G}\right) \nu_{Z(\vec{b})}(\vec{G})
$$

Because electron scattering may be strong, the kinematic model is not generally useful for the quantitative modeling of RHEED. In particular, for large grains, multiple scattering between states on the Ewald sphere may be important; this is a dynamical scattering process which will renormalize the kinematic scattering amplitudes. This effect will be most important for inner reflections where the coupling between scattered electron beams is strongest. For example, in the two beam case for a randomly oriented poly crystal, Cowley ${ }^{7}$ reports that the ratio of intensities between dynamical and kinematical scattering could be well represented by the equation

$$
I_{\text {dynamical }} / I_{\text {kinematic }}=F_{G}^{-1} \int_{0}^{F_{G}} d x J_{0}(2 x),
$$

where $F_{G}$ is $\nu(G) \lambda h / 4 \pi, \nu(G)$ is the electronic form factor for the reciprocal lattice vector $G, \lambda$ is the electron wavelength, and $h$ is the film thickness. Experiments by Horstmann and Meyer ${ }^{8}$ on aluminum found good agreement between this equation and experimental intensities, except for strong inner reflections like (400) and (222). While the films we are interested in are not randomly oriented, the crystals are sufficiently small that multiple scattering will occur between separate crystals, causing the dynamical intensities to add incoherently like in the randomly oriented films. Consequently, to first order, we expect that this effect will introduce a set of effective electron scattering potentials $\left\{V_{\vec{G}}^{\text {eff }}\right\}$ which will modify the relative intensities of different diffraction spots, and have little effect upon their shapes. Our present model ignores divergence or convergence of the incident beam which will introduce further spot broadening. Also ignored are inelastic events caused by scattering from surface plasmons and core level electronic excitations.

Often, for a RHEED pattern, a small number of reciprocal lattice vectors contribute to the intensity observed on the screen; thus a set of only a few $3 \times 3$ matrices $\mathbf{A}_{\vec{G}}$ are required to calculate the RHEED pattern. Calculation and inversion of $3 \times 3$ matrices requires negligible time and storage; so ultimately, the time required to calculate the predicted RHEED pattern is linear in the number of discrete pixels that have been chosen to be displayed on the diffraction screen. This permits real-time exploration of the effects of grain size and texture variation upon the RHEED diffraction pattern.

\section{APPLICATION TO POLYCRYSTALLINE MgO}

To demonstrate application of our model, we consider the RHEED pattern from small-grained, well-textured $\mathrm{MgO}$, a material that can be grown using ion-beam assisted deposition techniques. ${ }^{9} \mathrm{MgO}$ forms a Bravais lattice in the rock salt form with a lattice constant $a=0.42 \mathrm{~nm}$; the reciprocal lattice vectors of the perfect crystal satisfy

$$
G=l \frac{2 \pi}{a} \hat{x}+m \frac{2 \pi}{a} \hat{y}+n \frac{2 \pi}{a} \hat{z}
$$

$l, m, n$ all odd or all even integers.

The kinematic scattering potentials $V_{\vec{G}}$ needed to evaluate our model are related to the electronic form factors by

$$
V_{\vec{G}} \propto \begin{cases}\nu_{M g}(\vec{G})+\nu_{O}(\vec{G}) & l, m, n \text { even } \\ \nu_{M g}(\vec{G})-\nu_{O}(\vec{G}) & l, m, n \text { odd }\end{cases}
$$

To mimic real experimental configurations in molecular beam epitaxy, we consider RHEED from a polycrystalline sample of $\mathrm{MgO}$ whose normal points down, e.g., in the $-\vec{z}$ direction. The incident beam energy is taken to be $20 \mathrm{keV}$ and its direction of incidence to be $\hat{e}=\hat{x} \cos \theta_{\text {inc }}+\hat{z} \sin \theta_{\text {inc }}$, where $\theta_{\mathrm{inc}}=1.2^{\circ}$. The diffraction screen is placed normal to the $\hat{x}$ direction. Beams which scatter into the upper hemisphere, $(\hat{z} \bullet \hat{n})>0$, are directed into the wafer and so do not appear on the diffraction screen. In Fig. 1 we present a series of simulated diffraction patterns from well-textured smallgrained $\mathrm{MgO}$ with finite penetration depth. The through beam is not shown but would appear directly above the brightest spot (Miller index 00 $\overline{2}$ ) and equidistant from the spot directly beneath (Miller index $00 \overline{4}$ ).

In Fig. 1(a) we have set $\Delta \omega_{x}=\Delta \omega_{y}=\Delta \phi_{z}=0$ and $L_{g}$ $=h=4 \mathrm{~nm}$. The spots are circular and their "sharpness" (as measured by the rate that the spot intensities decay from their maxima) is proportional to the grain size. In Fig. 1(b) we have decreased the penetration depth to $1 \mathrm{~nm}$ without changing the lateral grain size or permitting any distribution in texture. The aspect ratio of the spots reciprocates the change in grain shape: as the grain height decreases the spot height increases. In Fig. 1(c) we have added a finite texture distribution to the polycrystalline $\mathrm{MgO}$ by increasing $\Delta \phi_{z}$ to $6^{\circ}$ and $\Delta \omega_{y}$ to $3^{\circ}$. Because $\hat{z}$ and $\hat{y}$ lie approximately tangent to the Ewald sphere for the roughly forward scattered electrons contributing to the intensity of these spots, texture rotations about these axes can only affect the relative intensities of the diffraction spots. Finally, in Fig 1(d), we have increased $\Delta \omega_{x}$ to $3^{\circ}$. The spots are seen to tip in response to the distribution in texture about the direction of the incident beam. If the lateral grain size and grain height were equal [see Fig. 1(a)], a distribution in texture about the $\hat{x}$ axis would smear the spots in circular arcs about the through beam. However, because the thickness and lateral size of the grains are unequal, the inclination and shape of the diffraction spots depend on $\Delta \omega_{x}, L_{g}$, and $h$ in a more complicated fashion.

\section{RHEED INTENSITY ANALYSIS}

All information about the grain size, in-plane orientation distribution, and texture distribution in a film is contained in the shapes and relative intensities of the diffraction spots seen on the RHEED screen. Figure 2 summarizes the qualitative dependence of spot shape and intensity upon the grain size, in-plane orientation distribution, and grain texture. These dependencies can be easily quantified. From Eq. (23) 

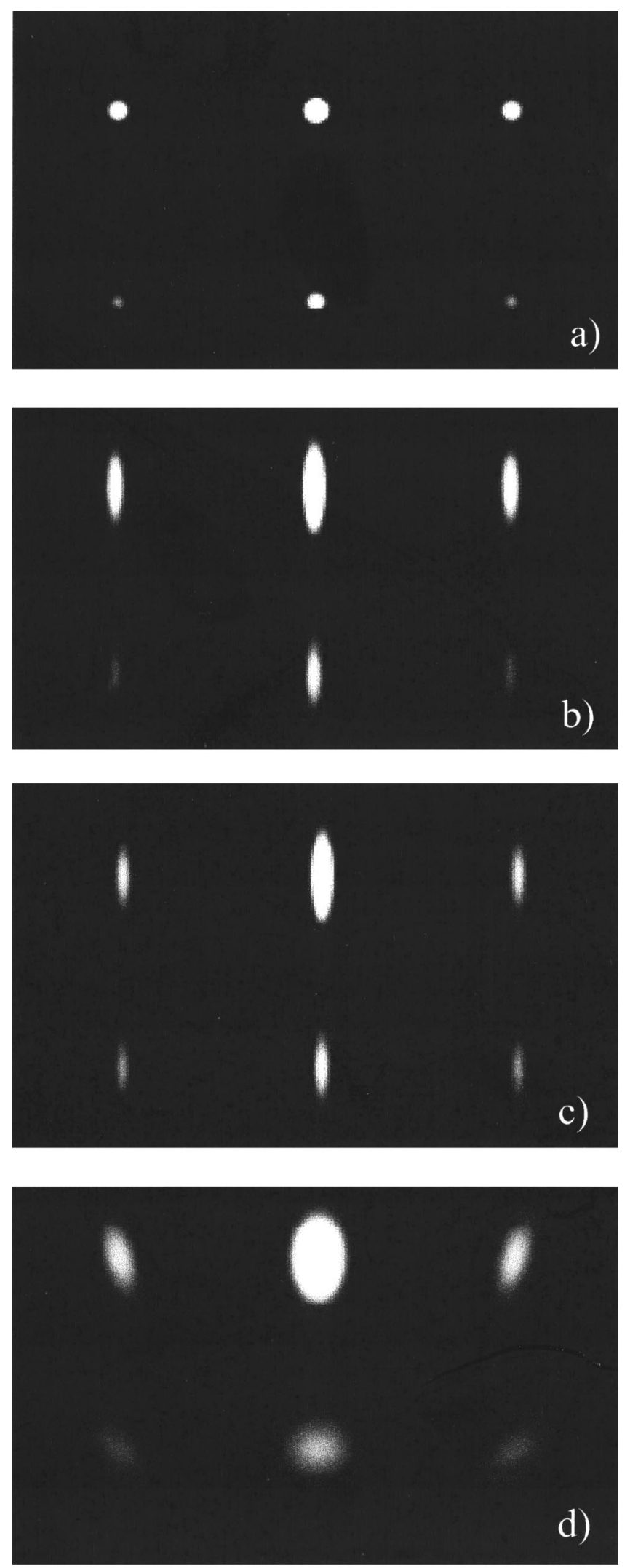

FIG. 1. Simulated RHEED patterns from polycrystalline $\mathrm{MgO}$ with finite grain size $L_{g}$, effective electron penetration depth $h$, and narrow grain orientation distributions, where the standard deviation of the distributions around the $x, y$, and $z$ axes are designated as $\Delta \omega_{x}, \Delta \omega_{y}, \Delta \phi_{z}$, respectively; (a) $\Delta \omega_{x}=\Delta \omega_{y}=\Delta \phi_{z}=0$ and $L_{g}=h=4 \mathrm{~nm}$; (b) same as (a) except $h$ $=1 \mathrm{~nm}$; (c) same as (b) except $\Delta \phi_{z}=6^{\circ}$ and $\Delta \omega_{y}=3^{\circ}$; (d) same as (c) except $\Delta \omega_{x}=3^{\circ}$.

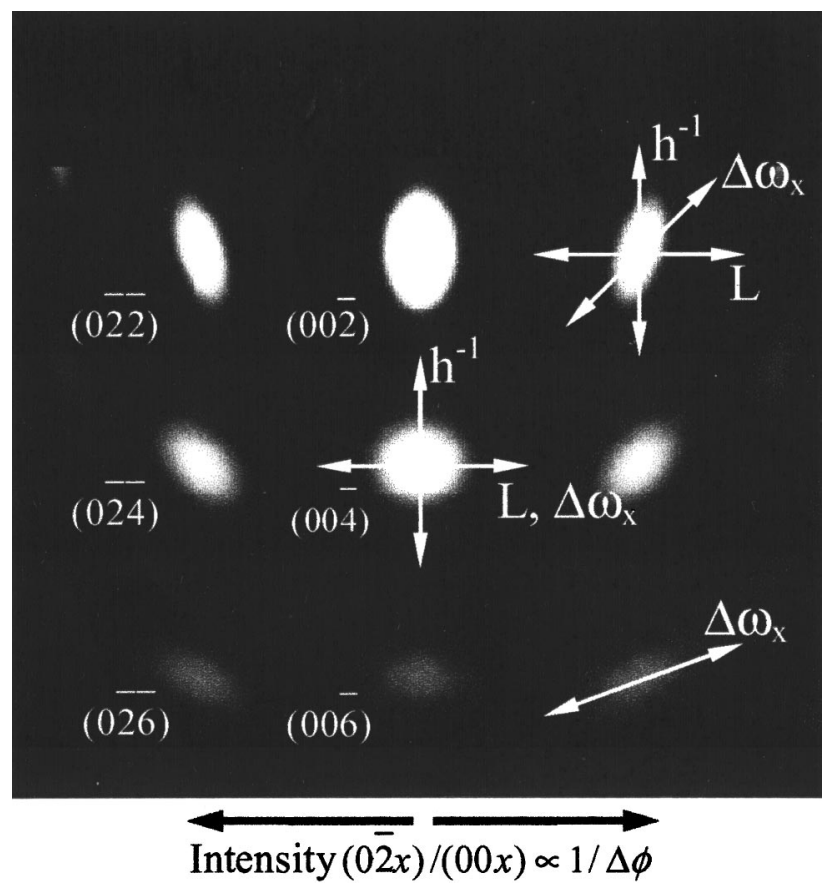

FIG. 2. Simulated RHEED pattern of $20 \mathrm{keV}$ electrons at $1.2^{\circ}$ grazing incidence along (001) [100] from well-textured polycrystalline $\mathrm{MgO}$ with lateral grain size $L_{g}=4 \mathrm{~nm}$, electron penetration depth $h=1 \mathrm{~nm}$, and grain orientation distributions with standard deviations $\Delta \omega_{x}=3^{\circ}, \Delta \omega_{y}=3^{\circ}$, and $\Delta \phi_{z}=6^{\circ}$, about the $x, y$, and $z$ axes. The qualitative effects of these parameters upon the RHEED spot shapes and relative intensities are indicated.

it is clear that in our model the RHEED intensity of a diffraction spot (labeled by the reciprocal lattice vectors $\vec{G}$ ) is described by an exponential of a quadratic form. In the limit of narrow diffraction spots we can write

$$
\begin{aligned}
\sum_{\vec{G}} \mid & \left.V_{\vec{G}}\right|^{2} \sqrt{\operatorname{det} \mid \mathbf{A}_{\vec{G}}} \mid \exp \left[-\frac{1}{2}(\vec{G}-k(\hat{n}-\hat{e}))^{T}\right. \\
& \left.\times \mathbf{A}_{\vec{G}}(\vec{G}-k(\hat{n}-\hat{e}))\right] \\
\propto & \sum_{\vec{G}}\left|V_{\vec{G}}\right|^{2} \sqrt{\operatorname{det} \mid \mathbf{A}_{\vec{G}}} \mid \exp \left[-\frac{1}{2}\left(\vec{G}-k\left(\hat{n}_{G}-\hat{e}\right)\right)^{T}\right. \\
& \left.\times \mathbf{A}_{\vec{G}}\left(\vec{G}-k\left(\hat{n}_{G}-\hat{e}\right)\right)\right] \exp \left(-\frac{1}{2} \vec{q}^{T} \mathbf{A}_{\vec{G}} \vec{q}\right),
\end{aligned}
$$

where we have separated the maximum in scattering intensity from the functional dependence of the scattering as it decays away from its maximum. The direction of maximum scattering intensity occurs in the direction $\hat{n}_{G}$, which satisfies

$$
\mathbf{A}_{\vec{G}}\left(\vec{G}-k\left(\hat{n}_{G}-\hat{e}\right)\right) \propto \hat{n}_{G} .
$$

The vector $\vec{q} \simeq k \vec{n}-k \vec{n}_{G}$ is restricted to the plane tangent to the Ewald sphere at $k \hat{n}_{G}$, e.g. $\hat{n}_{G} \bullet \vec{q}=0$. Contours of constant scattering intensity are ellipses and are described by

$$
\vec{q}^{T} \mathbf{A}_{\vec{G}} \vec{q}=\lambda_{+}\left(\hat{q}_{+}^{T} \vec{q}\right)^{2}+\lambda_{-}\left(\hat{q}_{-}^{T} \vec{q}\right)^{2}=\text { const, }
$$

where $\hat{q}_{+}$and $\hat{q}_{-}$are the principal axes of the ellipse and $\lambda_{+}$ and $\lambda_{-}$are the eigenvalues of the Hessian matrix of the diffraction intensity at the local diffraction intensity maximum. Large eigenvalues are characteristic of "sharp" dif- 
TABLE I. Quantitative characterization of the relative intensity, aspect ratio, angle of inclination, and intensity curvature of the simulated RHEED spots in Fig. 2. Spots are labeled by their reciprocal lattice vector.

\begin{tabular}{lcccc}
\hline \hline $1 \mathrm{~m} \mathrm{n}$ & $\begin{array}{c}\text { Relative } \\
\text { intensity }\end{array}$ & $\begin{array}{c}\text { Aspect } \\
\text { ratio }\end{array}$ & $\begin{array}{c}\text { Inclination } \\
\text { angle }\end{array}$ & $\begin{array}{c}\text { Curvature } \\
\left(\mathrm{nm}^{2}\right)\end{array}$ \\
\hline $0 \quad 0-2$ & 1.000 & 1.68 & $0.0^{\circ}$ & 8.15 \\
$0-2-118$ & 2.25 & $15.9^{\circ}$ & 7.93 \\
$0-2-2$ & 0.118 & 2.25 & $-15.9^{\circ}$ & 7.93 \\
$0 \quad 0-4$ & 0.078 & 1.11 & $90.0^{\circ}$ & 4.37 \\
$0 \quad-4$ & 0.035 & 1.65 & $43.4^{\circ}$ & 4.29 \\
$0-2-4$ & 0.035 & 1.65 & $-43.4^{\circ}$ & 4.29 \\
\hline \hline
\end{tabular}

fraction spots, so that the major axis of the ellipses of constant intensity will lie along the eigenvector with the smaller eigenvalue. If the eigenvalues of the Hessian matrix are equal, then the diffraction spot will be circular.

In practice, rather than solve Eq. (28) for the point of maximum intensity, it is easier to calculate the diffraction pattern and simply search for the local maxima. Once the
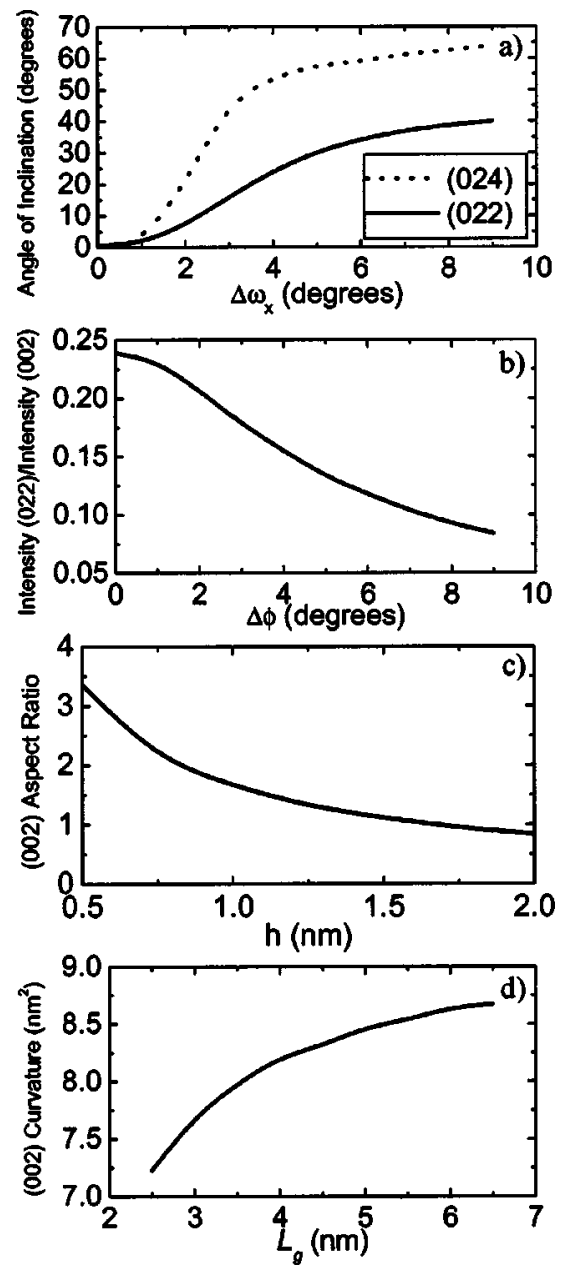

FIG. 3. Unless indicated otherwise, film parameters are as defined in Fig. 2: (a) angle of inclination of the $(0 \overline{2} \overline{4})$ and $(0 \overline{2} \overline{2})$ order diffraction spots as a function of the texture distribution width about the beam axis $\left(\Delta \omega_{x}\right)$; (b) relative intensity of the $(0 \overline{2} \overline{2})$ spot to that of the $(00 \overline{2})$ spot as a function of the distribution width of in-plane orientations in the film $\left(\Delta \phi_{z}\right)$; (c) aspect ratio of the $(00 \overline{2})$ spot as a function of scattering depth $h$; and (d) logarithmic curvature of the $(00 \overline{2})$ spot as a function of characteristic grain size $L_{g}$. maximum is known, fitting the spot for the principal axes and eigenvalues is accomplished by standard methods of linear algebra. Experimental data may also be quantified in this manner, but only if care has been taken not to saturate the detector when recording the RHEED pattern.

We have chosen to quantitatively characterize the diffraction spots in terms of four variables:

(1) relative intensity [see Eq. (28)],

(2) ellipse aspect ratio $\left(\sqrt{\lambda_{+} / \lambda_{-}}\right)$,

(3) ellipse inclination $\left(\arccos \left(\hat{z}^{T} \hat{q}_{-}\right)\right)$, and

(4) intensity curvature $\left(\sqrt{\lambda_{+} \lambda_{-}}\right)$.

Sample results for the quantitative characterization of the diffraction spots in Fig. 2 are presented in Table I.

Figure 3 demonstrates the sensitivity of the RHEED pattern to changes in grain size, electron scattering depth, and texture distribution. In each case the default values for the parameters are that of the RHEED pattern in Fig. 2. Figures $3(\mathrm{a})$ and 3(b) suggest that if spot inclination and relative spot intensity can be measured to within $5^{\circ}$ and $2 \%$, respectively, then the distributions in texture and in-plane orientation can be measured to within $1^{\circ}$. From Fig. 3(c), if the penetration depth is on the order of $1 \mathrm{~nm}$, the ability to measure spot aspect ratio to within $20 \%$ will allow one to determine the penetration depth to within a few angstroms. Finally, Fig. 3(d) indicates that if the curvature of the spot intensity can be measured to within a few percent then the characteristic lateral grain size can be determined to within a fraction of a nanometer.

\section{CONCLUSIONS}

We have presented a kinematic model for simulating RHEED from well-textured polycrystalline films which is able to incorporate the effects of finite grain size and electron penetration depth. We have also described a method to quantify RHEED patterns from such films in terms of the relative intensities, shapes, inclinations, and sharpness of the diffraction spots and shown how these parameters can be quantitatively related to lateral grain size, electron penetration depth, and distributions in crystallographic texture. Lateral grain size, penetration depth, and distribution in texture about the direction of the incident beam each affect the shape, sharpness, and inclination of the individual diffraction spots in a manner which will be well fit by our model. Distributions in texture about axes perpendicular to the incident beam (including in-plane orientation) affect the relative intensities of the diffraction spots and are more difficult to fit because the proper dynamic scattering potentials $V_{\vec{G}}$ also contribute to this effect but are not included in our model. However, the ability to monitor the time evolution of texture distributions about these axes is well within the capabilities of our model and sensitivity studies performed above on small grained polycrystalline $\mathrm{MgO}$ suggest that differences in lateral grain size as small as a few angstroms, and differences in texture distribution widths as small as $1^{\circ}$ can be easily detected with RHEED. 


\section{ACKNOWLEDGMENTS}

This work was supported by the DARPA Virtual Integrated Prototyping program and ARO MURI on "Engineering Microstructural Complexity in Ferroelectric Thin Films," Grant No. DAAD 19-01-1-0517. One of us (R.T.B.) is grateful for support from the Intel Foundation.

${ }^{1}$ S. Andrieu and P. Frechard, Surf. Sci. 360, 289 (1996).

${ }^{2}$ D. Litvinov, T. O'Donnell, and R. Clarke, J. Appl. Phys. 85, 2151 (1999).

${ }^{3}$ D. Litvinov, J.K. Howard, S. Khizroev, H. Gong, and D. Lambeth, J. Appl. Phys. 87, 5693 (2000).
${ }^{4}$ B.K. Vainshtein, B.B. Zvyagin, and A.S. Avilov, in Electron Diffraction Techniques, edited by J.M. Cowley (Oxford University Press, Oxford, 1992), Vol. 1, pp. 3-4, 245-250.

${ }^{5}$ Z.L. Wang, Elastic and Inelastic Scattering in Electron Diffraction and Imaging (Plenum, New York, 1995), pp. 7 and 9.

${ }^{6}$ B.D. Cullity, Elements of X-ray Diffraction, 2nd ed. (Addison-Wesley, Reading, MA, 1978), pp. 520-522.

${ }^{7}$ J. M. Cowley, Diffraction Physics, 3rd ed. (Elsevier Science, Amsterdam, 1995), pp. 380-382.

${ }^{8}$ M. Horstmann and G. Meyer, Z. Phys. 182, 380 (1965).

${ }^{9}$ C.P. Wang, K.B. Do, M.R. Beasley, T.H. Geballe, and R.H. Hammond, Appl. Phys. Lett. 71, 2955 (1997). 